\title{
Retenção da informação e percepção da justiça por parte dos alunos em relação ao controlo disciplinar em aulas de educação física
}

\author{
Nuno Januário ${ }^{1}$ \\ António Rosado ${ }^{1}$ \\ Isabel Mesquita²
}

https://doi.org/10.5628/pcd.06.03.294

\author{
1 Faculdade de M otricidade H umana \\ Universidade Técnica de Lisboa \\ Portugal \\ 2 Faculdade de D esporto \\ Universidade do Porto \\ Portugal
}

\section{RESUMO}

O presente estudo pretendeu analisar a retenção e a percepção da justiça dos alunos em relação aos comportamentos de regulação e controlo disciplinar por parte do professor, nomeadamente, nas reacções dos professores aos comportamentos de indisciplina. A amostra foi constituída por 147 respostas dos alunos às intervenç̃̃es de regulação dos comportamentos de indisciplina, por parte do professor a partir de 30 aulas de Educação Física dos 5ㅇ, 7ㅇe e 9 o anos de escolaridade. No presente estudo foram utilizadas, como variáveis independentes, as características dos alunos: idade, género, ano de escolaridade, número de reprovações e classificação na disciplina de Educação Física. Foram utilizadas, como variáveis dependentes, a quantidade de informação transmitida pelo professor (extensão, número de ideias e densidade da informação), a natureza da informação (forma, objectivo, direcção), a reprodução dessa informação pelo aluno, ao nível quantitativo (extensão, número de ideias, densidade) e qualitativo (coerência) e a percepção de justiça dessas reacções. Os comportamentos de indisciplina dos alunos e de controlo do professor foram gravados em suporte audiovisual, tendo sido solicitado aos alunos, de seguida, através de entrevista, que reproduzissem a mensagem transmitida pelo professor e que se pronunciassem acerca da justiça da reacção do professor. As respostas dos alunos foram gravadas em registo áudio. As reacções dos professores foram comparadas com as reproduções dos alunos através de procedimentos quantitativos e qualitativos (análise de conteúdo). Os resultados obtidos sugerem que a retenção da informação variou em função da natureza das mensagens e não de acordo com as características dos alunos. A coerência esteve inversamente relacionada com a quantidade de informação (extensão e o número de ideias). Globalmente os alunos perceberam as reacções dos professores como justas, condição fundamental de aceitação das reacções dos professores aos comportamentos de indisciplina.

Palavras-chave: educação física, indisciplina, retenção de informação, coerência de informação, percepção de justiça.

\section{ABSTRACT \\ Student's Retention of Information and Justice Perceptions regarding Discipline Control}

The aim of the present study is to analyze students' retention and acceptance (perception of justice) of teachers' discipline control interventions, namely, teachers' reactions to misbehaviors. We observed 30 physical education lessons and analyzed 147 student's responses to misbehavior reactions. The independent variables were age, gender, school year, school reproofs and Physical Education marks. The dependant variables were the quantity of information on teacher instructions (extension, number of ideas and density of teacher' information) and the nature of the information (objective, form and direction), the reproduction of that information by the students, both in a quantitative (extension, number of ideas, density) and qualitative (coherency) approach. The perception of justice depending on the teacher's reaction was also studied. The student's misbehaviors and teacher's reactions were audio and video recorded and immediately afterwards students were asked (by interview) to reproduce the teacher's information, being also asked about the fairness of the reaction. This procedure was audiorecorded. The information given by the teacher and the student's answers were analyzed and compared through quantitative and qualitative procedures (content analysis). The results suggest that the retention of the information depends, generally, on its nature and not on the student's personal characteristics. The coherency of the information was inversely related with the quantity of information (number of transmitted ideas and its extension). Globally, the students perceived teachers reaction as fair, a basic condition to obtain students acceptance of teachers behaviors.

Key-words: physical education, retention of information, coherency of information, misbehaviors, perception of fairness. 


\section{INTRODUÇÃO}

Os comportamentos desviantes ou de indisciplina parecem figurar entre os que, mais frequentemente, apresentam uma relação negativa com as aprendizagens e com o clima da aula, sendo os comportamentos perturbadores dos alunos percebidos pelos professores como ameaças à criação de um clima pedagógico favorável e, consequentemente, às aprendizagens. A investigação subordinada à temática da indisciplina, em termos genéricos, tem sido estudada por autores como Sulzer-Azaroff e Mayer (30), Good e Brophy (17), Fontana (15), Estrela (9,10,11) e Alonso (1). No contexto do ensino da Educação Física e do Desporto diferentes estudos podem ser encontrados, tanto ao nível internacional $(5,20,23,29)$ como ao nível nacional $(4,16,18,22,25,26,27)$.

A eficácia das instruções de controlo e regulação comportamental, em particular, pode afectar, não só, a qualidade do controlo da classe, uma variável associada à eficácia pedagógica, mas, também, a qualidade da relação interpessoal e a prossecução de objectivos de formação pessoal e social $(12,13)$. As reacções dos professores aos comportamentos de indisciplina constituem, especificamente, episódios importantes na construção da qualidade da relação pedagógica e na regulação dos comportamentos $(10,11)$. A reacção a um incidente de indisciplina resulta da tomada de decisões que, para serem eficazes, devem considerar a ecologia da situação (8,31). Em função das características da situação, o professor reage aos comportamentos de indisciplina, no sentido de os regular, utilizando um conjunto diversificado de reacções, verbais e não-verbais, que vão, a título de exemplo, das mais brandas, como o aviso ou a repreensão, passando por ordens de interrupção do comportamento ou de realização do comportamento correcto, até ao afastamento temporário ou à exclusão da aula. Nas diferentes reacções de controlo disciplinar utilizadas pelo professor, a transmissão de informação ao aluno está sempre presente, podendo apresentar diferentes características. Essas reaç̧ões, constituídas por mensagens e instruções específicas, são percebidas pelos alunos de formas diversificadas quer no que se refere à retenção e compreensão $(6,7)$ quer no que se refere à sua aceitação. $A$ informação de controlo disciplinar emitida pelo professor integra diferentes unidades informativas, possibilitando, por parte dos alunos, diversos níveis de retenção, compreensão e aceitação das mensagens aí contidas, pelo que o estudo destas dimensões e das variáveis que as afectam pode favorecer aspectos importantes da comuni cação do professor. Sabe-se que a qualidade da informação transmitida resulta dum largo conjunto de variáveis $(2,12,19)$ mas, em última análise, depende do seu poder persuasivo, isto é, seu nível de compreensão, retenção e aceitação. Estes episódios instrucionais, de reacção a comportamentos inapropriados, devem ser estudados com particular atenção, uma vez que é de esperar que uma parte significativa das mensagens transmitidas pelo professor se perca entre o momento da emissão e o da recepção, ou que, no mínimo, seja alterada por diversos filtros perceptivos pessoais, perdendo, assim, parte da sua potencial eficácia pedagógica $(3,6$, 7, 18, 21, 24, 27).

A investigação das temáticas ligadas à retenção da informação, à sua compreensão e aceitação é reduzi$\mathrm{da}$, tendo sido, prioritariamente, dirigida para outros tipos de instrução, nomeadamente, o feedback pedagógico e a apresentação de tarefas motoras. Marques da Costa (21) estudou o impacto do feedback pedagógico na aprendizagem, tendo como objectivo conhe cer o efeito, nos alunos, da informação emitida pelo professor. A autora constatou que os alunos conseguiam relatar apenas parte da informação transmitida pelo professor (com uma coerência de cerca $70 \%$ ) e que o grau de coerência do relato não dependia do género, idade, tipos de memória, inteligência e habilidade motora inicial, mas sim das características do feedback, nomeadamente, da sua estrutura e extensão. A autora constatou, ainda, que os feedbacks interrogativos e descritivos tinham valores de coerência superiores aos prescritivos, colocando, os últimos, maiores problemas de retenção. Também Quina (24), estudando a retenção de informação no feedback, verificou que o nível médio de retenção dos alunos era de $56,7 \%$ e que os al unos relatavam com mais fidelidade os feedbacks descritivos áudio. Constatou, ainda, que as estruturas informacionais descritivas e pouco densas tiveram maior nível de retenção do que as estruturas prescritivas extensas. Breia e Rosado (3), no contexto do treino desportivo, estudando a retenção de informação em diversos tipos de instrução, verificou que os atletas evocaram 
$58,7 \%$ do total de ideias e que a retenção da informação não apresentava, genericamente, diferenças significativas, nem em função da natureza da informação transmitida nem das características dos atletas. Os feedbacks com informação prescritiva apresentaram valores médios de retenção de informação mais elevados, o que parece contrariar dados anteriores (21, 24). Em nenhum destes estudos se abordou a reacção aos comportamentos de indisciplina, pelo que as extrapolações baseadas na análise do feedback devem ser relativizadas.

Por outro lado, os estudos consultados, embora abordando a problemática da retenção de informação, não consideraram a questão da sua aceitação por parte dos alunos. Na realidade, o estudo do processo persuasivo presente nos diversos episódios instrucionais deve estender-se à sua aceitação pelos alunos, considerando a credibilidade e a adesão concedidas a essas instruções. No caso das reacções aos comportamentos de indisciplina, a aceitação está intimamente dependente da percepção do grau de justiça da reacção do professor. Flavier et al., (14) ao analisarem a dinâmica intrínseca do envol vimento colectivo em situações de conflito, chamam a atenção para os procedimentos de gestão da percepção de justiça. No entanto, não foi possível encontrar qualquer outro trabal ho que abordasse a temática referida de uma forma mais específica. Sabe-se, contudo, que as concepções de ensino, os valores pedagógicos e as crenças dos professores e dos alunos afectam a percepção de adequação e justiça dos comportamentos adoptados (26).

A dinâmica dos processos de persuasão instrucional (retenção, compreensão e aceitação da informação) carece de estudos em situações de regulação e controlo disciplinar, não existindo suficiente evidência científica acerca da relação entre as características da informação instrucional e a retenção dessa informação. Também não é possível destacar a influência de variáveis como as características dos alunos sobre este processo. É possível que variáveis como a idade, o género, o nível de escolaridade, o nível de sucesso escolar e o nível de sucesso na disciplina de Educação Física possam influenciar a capacidade de retenção da informação, presente nas reacções dos professores aos comportamentos de indisciplina, bem como a aceitação dessas reacções por parte dos alunos.
O presente estudo pretendeu analisar a retenção e a aceitação (avaliada através da percepção da justiça da reacção), pelos alunos, da informação transmitida pelo professor em situações de reaç̧ão aos comportamentos de indisciplina.

Para o efeito, pretendeu-se responder às seguintes questões: Qual o nível de retenção das informações transmitidas? Qual o nível de aceitação (percepção de justiça) dessas reacções? Que factores ligados às características da informação influenciam a retenção? Que factores ligados às características dos alunos (idade, género, nível de escolaridade, nível de sucesso escolar e nível na disciplina de Educação Física) afectam a retenção? Estas questões parecemnos, ainda, particularmente importantes do ponto de vista da intervenção profissional, na medida em que podem auxiliar a definir estratégias de instrução facilitadoras da gestão de situações de indisciplina, bem como a reflexão acerca do valor das informações transmitidas e dos processos comunicativos.

\section{MATERIAL E MÉTODOS}

Sujeitos

Foi observado um total de 30 sessões de Educação Física em 15 turmas ( 2 sessões por turma), sendo 5 turmas de 5o ano, 5 turmas de 70 ano e 5 turmas de 9o ano de escolaridade, em escolas da área da Grande Lisboa (António Gedeão - Almada, Mouzinho da Silveira - Barreiro e Maria Veleda Loures). A amostra foi constituída pela totalidade das respostas dos alunos $(n=147)$ referentes aos episódios de reacção dos professores aos comportamentos de indisciplina, envolvendo um total de 87 alunos. O género masculino apresentou 59 alunos e o género feminino 28. No que respeita aos anos de escolaridade, o 70 ano apresentou 39 alunos, o 5o ano, 34 al unos e, o 9o ano, 14 alunos. A idade dos alunos variou entre os 9 e 15 anos. No que respeita ao número de repetências, 59 alunos nunca repetiram, 20 alunos repetiram uma única vez e 8 alunos repetiram o ano por duas ou mais vezes. Em relação à classificação final obtida na disciplina de Educação Física, 33 alunos obtiveram nível 3, 49 alunos obtiveram nível 4 e 6 alunos obtiveram nível 5 .

Variáveis

Neste estudo foram constituídos os seguintes grupos de variáveis: 
Variáveis caracterizadoras dos alunos: idade, género, ano de escolaridade, número de reprovações, classificação na disciplina de educação física.

Variáveis relacionadas com a transmissão da informação pelo professor: em termos quantitativos, a extensão da informação (número de palavras transmitidas), número de ideias (número de informações diferentes contidas nos episódios de reacção ao comportamento dos alunos) e densidade informativa (razão entre o número de ideias e o número de palavras utilizadas para as emitir). No que se refere à natureza da informação, a forma da informação (auditiva, visual e mista), o objectivo da informação (informação avaliativa, prescritiva, descritiva, interrogativa e mista) e a direcção da informação (informação individual e informação ao grupo). No caso da informação visual contabilizou-se a extensão da informação visual (número de unidades de informação visual) e o número de ideias com expressão exclusivamente visual. A informação mista, por seu lado, contabilizou as informações auditivas e visuais presentes na mesma intervenção.

Para a descrição e categorização da informação transmitida pelo professor, utilizámos um sistema de categorias designado Sistema de A nálise da Informação Transmitida na R eacção a Comportamentos Inapropriados (abreviadamente, SAITRCI). O sistema resultou de uma adaptação do Sistema de 0 bservação do Feedback Pedagógico, desenvolvido por Sarmento et al., (28). As categorias foram objecto de definição operacional e a aplicação do sistema envolveu treino prévio de observação e codificação.

Variáveis relacionadas com a informação reproduzida e a percepção de justiça por parte dos alunos: extensão da informação reproduzida (número de palavras usadas pelos alunos), número de ideias reproduzidas, densidade (razão entre o número de ideias e o número de palavras usadas para as expressar), coerência (razão entre o número de ideias concordantes entre o professor e o aluno) e percepção de justiça (aval iação pelo aluno do grau de justiça da reacção do professor).

Fidelidade do sistema de medida

Foi testada a fidelidade inter-codificador e intra-codificador. A pós treino do sistema, com base na análise de uma sessão de 50 minutos, a fidelidade intracodificador envolveu duas aplicações com o intervalo de dez dias sobre uma amostra de $20 \%$ do protocolo de informação recolhida do professor e do aluno. A fidelidade inter-observadores utilizou 2 codificadores que codificaram independentemente a mesma informação. A obtenção das medi das relativas à extensão da informação e número de ideias, não obtidas directamente pelo sistema de categorias definido, foi, também, objecto de aval iação da fidel idade inter e intra-codificador. Utilizou-se a percentagem de acordos para medir a fidelidade. Os níveis de acordo foram 95\% para a fidelidade inter-observador e $100 \%$ para a fidelidade intra-observador.

\section{Procedimentos}

Obtido o consentimento de professores e alunos e explicitados os objectivos do trabalho junto de professores e alunos, as sessões foram gravadas, na íntegra, em registo vídeo e áudio, com destaque para as instruções do professor relacionadas com as reacções de regulação e controlo disciplinar. Uma câmara vídeo, em grande angular, seguindo a total idade da turma, foi estrategicamente colocada num dos topos do espaço desportivo. O professor transportava um microfone de lapela com transmissão sem fio para gravação das suas instruções. Após cada reacção a um comportamento de indisciplina, pelo professor, o aluno foi abordado pelo investigador e foi realizada uma entrevista constituída por duas questões (uma em que se pedia ao aluno para repetir o que o professor tinha dito e outra em que se perguntava ao aluno se considerava justa a reacção do professor). Nenhuma reaç̧ão gerou cargas emocionais consideradas particularmente intrusivas, tendo-se previsto que nenhum aluno fosse inquirido em situação de descompensação emocional. Procedeu-se, de seguida, à transposição para protocolo escrito de todas as intervenções dos professores (relativamente às instruções de controlo disciplinar) e dos alunos, sendo essa informação submetida a anál ise de conteúdo. As unidades de registo obtidas constituíram a base da análise da informação e da comparação entre a informação transmitida e a informação evocada.

\section{Tratamento dos dados}

Os dados foram descritos através do cálculo de frequências de resposta, das médias, do desvio-padrão e dos valores mínimos e máximos. A normalidade 
das variáveis foi verificada através dos testes estatísticos Kolmogorov-Smirnov e Shapiro-Wilk. As provas estatísticas comparativas utilizadas, em consequência das análises anteriores, que não verificaram os requisitos de normalidade e homogeneidade das variâncias, foram as provas $U$ de $M$ ann-W hitney, Kruskal-Wallis e Binomial, em função da natureza dos problemas de pesquisa. Recorreu-se, ainda, à correlação de Spearman para verificar o grau de associação das variáveis. Para todos os testes realizados utilizámos um nível de significância de $p \leq 0.05$.

\section{APRESENTAÇÃO E DISCUSSÃO DOS RESULTADOS Coerência da informação}

Mais de metade dos alunos (56,5\%), apresentou um valor de coerência de $100 \%$, ou seja, relatou, coerentemente, todas as ideias veiculadas pelo professor. Os restantes alunos (43,5\%), apresentaram dificuldades em reproduzir as ideias do professor (em parte ou na totalidade), o que significa uma perda substantiva na recuperação de informação nestes momentos instrucionais. Em média, os val ores de coerência foram de $68,4 \%$. Este resultado da coerência de informação restituída é superior ao encontrado por Quina (24), 56,7\%, e aproximado do encontrado por Marques da Costa (21), (71\%), ambos em episódios instrucionais de feedback pedagógico. No entanto, o estudo de Marques da Costa real izou-se em condições de interferência contextual reduzida, o que pode explicar os val ores elevados encontrados nesse estudo.

O quadro 1 mostra as correlações entre a coerência e o número de ideias transmitidas bem como a correlação da coerência com a extensão da informação transmitida pelo professor.

Quadro 1. Correlação entre coerência e extensão da informação e coerência e número de ideias transmitidas.

\begin{tabular}{|l|c|c|c|c|}
\hline & \multicolumn{2}{|c|}{$\begin{array}{c}\text { Extensão da } \\
\text { informação }\end{array}$} & \multicolumn{2}{c|}{$\begin{array}{c}\text { Número de ideias } \\
\text { transmitidas }\end{array}$} \\
\hline & $\begin{array}{c}\text { Coeficiente de } \\
\text { Correlação }\end{array}$ & Sig & $\begin{array}{c}\text { Coeficiente de } \\
\text { Correlação }\end{array}$ & Sig \\
\hline Coerência &,- 283 &, 001 &,- 537 &, 000 \\
\hline
\end{tabular}

A coerência, quando relacionada com a extensão da informação, apresenta uma correlação fraca e negativa. Estes resultados sugerem que, quando a informação é menos extensa, a coerência aumenta, embora esta associação seja, na realidade, fraca.

No que se refere à correlação entre a coerência e o número de ideias transmitidas constatamos a existência de uma correlação moderada, também de sentido negativo, evidenciando que a coerência aumenta quando diminui o número de ideias transmitidas. Destaca-se, também, que a coerência da informação está mais associada ao número de ideias transmitidas do que à extensão da informação, sugerindo que o número de ideias transmitidas tem maior valor preditivo sobre a coerência da informação.

Sublinhamos, assim, que a coerência está inversamente relacionada tanto com a extensão da informação como com o número de ideias transmitidas.

Influência das características da informação sobre a retenção

O bjectivo da informação transmitida Quando considerámos os diversos tipos de objectivos da informação transmitida e as suas relações com as variáveis referentes à reprodução e retenção da informação, verificámos que apenas foram encontradas diferenças significativas para as variáveis densi dade e coerência (Quadro 2). 
Quadro 2. Comparação das variáveis ideias reproduzidas, extensão reproduzida, densidade e coerência da informação em função do objectivo da informação transmitida.

\begin{tabular}{|c|c|c|c|c|c|c|c|c|c|c|c|}
\hline & & Ideias re & duzidas & $\begin{array}{l}\text { Ext } \\
\text { repr }\end{array}$ & $\begin{array}{l}\text { são } \\
\text { Izida }\end{array}$ & & Densida & & & Coerênc & \\
\hline Objectivo & $\mathrm{n}$ & média & $(p-K W)$ & média & $(p-K W)$ & média & (p-KW) & (p-MW) & média & (p-KW) & (p-MW) \\
\hline Prescritivo & 69 & 1,25 & \multirow{4}{*}{0,716} & 4,83 & \multirow{4}{*}{0,39} & 31,2 & \multirow{4}{*}{0,035} & \multirow{4}{*}{$\begin{array}{l}\text { Pres/ } \\
\text { Inter } \\
=0,006\end{array}$} & 71,5 & \multirow{4}{*}{0,02} & \multirow{4}{*}{$\begin{array}{c}\text { Pres/ } \\
\text { Misto } \\
=0,012 \\
\text { Inter } / \\
\text { Misto } \\
=0,003\end{array}$} \\
\hline Descritivo & 15 & $1,4 ?$ & & 6,40 & & 24,1 & & & 55,6 & & \\
\hline Interrogativo & 40 & 1,13 & & 5,70 & & 23,4 & & & $? 7,1$ & & \\
\hline Misto & 23 & 1,39 & & 6,00 & & 28,8 & & & 52,2 & & \\
\hline
\end{tabular}

Legenda: Kruskal - Wallis (KW) e U de Mann-Whitney (MW).

A informação transmitida com objectivos exclusivamente prescritivos apresentou um val or médio, de densidade, superior ao da informação com objectivos interrogativos $(p=0.006)$. Por outro lado, as diferenças do valor médio da coerência, em função dos objectivos da informação, revelaram-se significativas quando a comparação foi entre a informação com objectivo prescritivo e a informação com objectivo misto $(p .=0,012)$ e entre a informação com objectivo interrogativo e a informação com objectivo misto $(p=0,003)$.

Comparando os resultados da presente pesquisa com os de Marques da Costa (21), podemos constatar que os valores são semelhantes para a informação com objectivo prescritivo e interrogativo. Neste trabal ho, os valores médios foram de $71,5 \%$ para a informação com objectivo prescritivo e de $77 \%$ para a informação de cariz interrogativo, apresentando valores de coerência de $71 \%$ e $80 \%$, respectivamente. A diferença significativa dos níveis de coerência entre a informação com objectivos mistos e a informação com objectivos prescritivos ou interrogativos, devese, em nossa opinião, ao facto da informação com objectivos mistos consistir, usualmente, em intervenções mais extensas, logo com maior dificuldade de retenção.
Forma da informação transmitida A forma de transmissão da informação mostrou influenciar a reprodução e a retenção da informação. Como se pode constatar no quadro 3 foram encontradas diferenças significativas na densidade $(p=0,002)$ e na coerência, em função da forma como esta era transmitida $(p=0,047)$. 
Quadro 3. Comparação das variáveis ideias reproduzidas, extensão reproduzida, densidade e coerência da informação em função da forma da informação.

\begin{tabular}{|c|c|c|c|c|c|c|c|c|c|c|c|}
\hline \multirow[b]{2}{*}{ Forma } & \multirow[b]{2}{*}{$n$} & \multicolumn{2}{|c|}{$\begin{array}{c}\text { Ideias } \\
\text { reproduzidas }\end{array}$} & \multicolumn{2}{|c|}{$\begin{array}{c}\text { Extensão } \\
\text { reproduzida }\end{array}$} & \multicolumn{3}{|c|}{ Densidade } & \multicolumn{3}{|c|}{ Coerência } \\
\hline & & média & $(p-K W)$ & média & $(p-K W)$ & média & $(p-K W)$ & (p-MW) & média & $(p-K W)$ & (p-MW) \\
\hline Auditivo & 65 & 1,23 & \multirow{3}{*}{0,799} & 4,95 & \multirow{3}{*}{0,115} & 28,52 & \multirow{3}{*}{0,002} & \multirow{3}{*}{$\begin{array}{l}\text { Auditivo/ } \\
\text { Visual=0,029 } \\
\text { Auditivo/ Misto } \\
=0,011 \\
\text { Visual/Misto } \\
=0,003\end{array}$} & 74,05 & \multirow{3}{*}{0,046} & \multirow{3}{*}{$\begin{array}{l}\text { Auditivo/ } \\
\text { Misto } \\
=0,033\end{array}$} \\
\hline Visual & 15 & 1,20 & & 3,20 & & 49,05 & & & 80,00 & & \\
\hline Misto & 67 & 1,29 & & 6,01 & & 25,93 & & & 62,04 & & \\
\hline
\end{tabular}

Legenda: Kruskal- Wallis (KW) e U de Mann - Whitney (MW)

Os dados revelaram diferenças significativas no que respeita à densi dade informativa em função da forma da informação. Estas diferenças foram significativas na comparação entre as três formas de informação, tendo a forma visual apresentado o valor mais elevado e a forma mista o valor mais baixo. No que respeita à coerência, a informação transmitida pelo canal auditivo registou um valor médio de coerência de $74 \%$, enquanto que a informação transmitida de uma forma mista apresentou um valor médio de $62 \%$. Esta diferença revelou-se, também, significativa $(p=0,033)$.

Os valores por nós encontrados são muito próximos dos valores encontrados por Marques da Costa (21), com val ores de coerência de $75 \%$ (auditivo) e $60 \%$ (misto). Por seu turno, Quina (24) encontrou valores inferiores, $62 \%$ e $52 \%$ (auditivo e misto, respectiva mente). Na realidade, a maior extensão da informação do tipo misto, associada à maior complexidade deste tipo de informação, pode explicar estes resultados.

Direcção da informação transmitida

A direcção da informação transmitida foi prioritariamente ( $71,4 \%$ ) individual e em $28,6 \%$ das situações dirigida a um pequeno grupo (Quadro 4). Esta diferença de val ores parece-nos perfeitamente justificável porquanto, na maior parte dos comportamentos de indisciplina observados, apenas um aluno esteve envolvido. $O$ estudo permitiu, ainda, constatar que a direcção da informação não exerce influência sobre nenhuma das variáveis de reprodução e retenção da informação (Quadro 4).

Quadro 4: Comparação das variáveis ideias reproduzidas, extensão reproduzida, densidade e coerência da informação em função da direcção da informação.

\begin{tabular}{|c|c|c|c|c|c|c|c|c|c|}
\hline & & \multicolumn{2}{|c|}{ Ideias reproduzidas } & \multicolumn{2}{|c|}{ Extensão reproduzidas } & \multicolumn{2}{|c|}{ Densidade } & \multicolumn{2}{|c|}{ Coerência } \\
\hline Direcção & $\mathrm{n}$ & média & (p) & média & (p) & média & (p) & média & (p) \\
\hline Aluno & 105 & 1,31 & \multirow[t]{2}{*}{0,209} & 5,56 & \multirow[t]{2}{*}{0,626} & 28,78 & \multirow[t]{2}{*}{0,486} & $6 ?, 14$ & \multirow[t]{2}{*}{0,682} \\
\hline Grupo & 42 & 1,12 & & 5,02 & & 25,96 & & 71,43 & \\
\hline
\end{tabular}


Análise da informação em função das características dos alunos

No quadro 5 estão patentes os resultados da comparação das variáveis ideias reproduzidas, extensão reproduzida, densidade e coerência da informação, em função das características dos alunos.

A análise da informação reproduzida em função das características dos alunos evidenciou que não existiram diferenças significativas no que se refere ao número de ideias reproduzidas, à densidade informacional e à coerência. Contrariamente, Quina (24), verificou que a idade influenciou a coerência da informação relatada pelos alunos.
Variáveis como o género, o nível escolar (apreciado pelo número de repetências) e o nível a Educação Física (avaliado pela classificação na disciplina) não afectaram, também, nenhum dos indicadores de reprodução e retenção de informação. Tal reforça a ideia já enunciada por Marques da Costa (21) de que parecem ser as características das mensagens e não as características dos alunos a desempenharem o papel determinante na retenção da informação. Das variáveis referentes às características dos alunos, apenas a idade e o ano de escolaridade apresentaram diferenças significativas na extensão da informação reproduzida.

Quadro 5. Comparação das variáveis ideias reproduzidas, extensão reproduzida, densidade e coerência da informação em função das características dos alunos.

\begin{tabular}{|c|c|c|c|c|c|c|c|c|c|}
\hline \multirow[b]{2}{*}{ ANO } & \multicolumn{2}{|c|}{ Ideias reproduzidas } & \multicolumn{3}{|c|}{ Extensão reproduzida } & \multicolumn{2}{|c|}{ Densidade } & \multicolumn{2}{|c|}{ Coerência } \\
\hline & média & $(p-K W)$ & média & $(\mathrm{p}-\mathrm{KW})$ & $(p-M W)$ & média & $(p-K W)$ & média & $(p-K W)$ \\
\hline $5^{\circ}$ & 1,39 & \multirow{3}{*}{0,195} & 6,29 & \multirow{3}{*}{0,025} & \multirow{3}{*}{$\begin{array}{l}5^{\circ} / 7^{\circ}=0,017 \\
5^{\circ} / 9^{\circ}=0,029\end{array}$} & 25,75 & \multirow{3}{*}{0,129} & 70,83 & \multirow{3}{*}{0,839} \\
\hline 70 & 1,21 & & 4,97 & & & 31,24 & & $65,6 ?$ & \\
\hline $9^{\circ}$ & 1,28 & & 4,55 & & & 25,82 & & 70,14 & \\
\hline \multicolumn{10}{|l|}{ IDADE } \\
\hline 9 & 1 & \multirow{7}{*}{0,062} & 5 & \multirow{7}{*}{0,043} & \multirow{7}{*}{$\begin{array}{l}10^{\%} / 11^{\circ}=0.024 \\
10^{\circ} / 13^{\circ}=0.009 \\
10^{\circ} / 14^{\circ}=0.041 \\
11 / 15^{\circ}=0,030 \\
13 / 15^{\circ}=0,035\end{array}$} & 21,22 & \multirow{7}{*}{0,119} & 64,29 & \multirow{7}{*}{0,108} \\
\hline 10 & 1,62 & & $?, 08$ & & & 25,74 & & 75,64 & \\
\hline 11 & 0,94 & & 4,06 & & & 26,51 & & 78,13 & \\
\hline 12 & 1,38 & & 6 & & & 29,4 & & 51,39 & \\
\hline 13 & 1,33 & & 4,9 & & & 34,16 & & 58,35 & \\
\hline 14 & 1,09 & & 4,68 & & & 28,87 & & 71,97 & \\
\hline 15 & 1,33 & & 6,44 & & & 22,24 & & 88,89 & \\
\hline \multicolumn{10}{|l|}{ GÉNERO } \\
\hline Masculino & 1,25 & \multirow{2}{*}{0,62} & 5,07 & \multirow{2}{*}{0,129} & & 29,45 & \multirow{2}{*}{0,133} & 66,35 & \multirow{2}{*}{0,333} \\
\hline Feminino & 1,34 & & 6,29 & & & 24,02 & & 73,5 & \\
\hline \multicolumn{10}{|l|}{ CLASSIFICAÇÃO } \\
\hline 3 & 1,23 & \multirow{3}{*}{0,781} & 5,74 & \multirow{3}{*}{0,56} & & 26,72 & \multirow{3}{*}{0,246} & 72,4 & \multirow{3}{*}{0,647} \\
\hline 4 & 1,29 & & 5,2 & & & 29,29 & & 66,46 & \\
\hline 5 & 1,33 & & $5,6 ?$ & & & 27,86 & & 63,89 & \\
\hline \multicolumn{10}{|l|}{ REPETÊNCIAS } \\
\hline 0 & 1,25 & \multirow{3}{*}{0,952} & 5,3 & \multirow{3}{*}{0,21} & & 28,85 & \multirow{3}{*}{0.295} & 68,85 & \multirow{3}{*}{0,17} \\
\hline 1 & 1,27 & & 5,3 & & & 33,14 & & 65,42 & \\
\hline$>1$ & 1,29 & & 6,43 & & & 21,72 & & 86,9 & \\
\hline
\end{tabular}

Legenda: Kruskal- Wallis (KW) e U de Mann - Whitney (MW) 
Apenas na variável referente à extensão da informação foram encontradas diferenças significativas entre os al unos do 5o e do 70 ano de escolaridade $(p=$ $0,017)$ e entre os alunos do 5o e do 9o ano ( $p=$ 0,029 ), pertencendo os valores superiores ao grupo etário mais baixo.

Por seu turno, na análise desta mesma variável, em função da idade, verificaram-se diferenças significativas entre os alunos de 11 e 15 anos $(p=0,030)$, sendo que os mais velhos relataram, em geral, com maior número de palavras, a informação que o professor transmitiu; a mesma tendência foi registada entre os 13 e 15 anos ( $p=0,035)$. Excepção a esta constatação foi a idade de 10 anos na qual se verificaram os valores mais elevados, registando-se diferenças significativas com os 11 anos $(p=0,024), 13$ anos $(p=0,009)$ e 14 anos $(p=0,0041)$. Os resultados não evidenciam, no entanto, nenhum padrão consistente que permita associar a idade às características da informação reproduzida e à sua coerência.

\section{Percepção de justiça dos alunos acerca} das reacções de controlo disciplinar

De um total de 147 reacções de regulação e controlo disciplinar, 123 foram consideradas justas e 13 foram consideradas injustas. O el evado número de respostas consideradas justas impediu a análise da variabilidade da percepção de justiça em função das características dos alunos, trabal ho que consideramos necessário prosseguir em estudos futuros. Neste estudo constatámos que em 90,4\% dos episódios a reacção foi aval iada como justa, tendo os restantes 9,6\% sido percebidos como injustos. Em função dos valores apresentados podemos concluir, globalmente, que os alunos consideram que as reacções dos professores, em situação de regulação e controlo disciplinar, são justas, sendo, por essa via, elevados os níveis de aceitação dessa reacção. Estes elevados níveis de aceitação configuram uma avaliação do processo instrucional que pode ser entendido como adequado e são condição para garantir os efeitos persuasivos que deve possuir qualquer acto instrucional, em particular, os que envolvem comportamentos de indisciplina. O facto de a percepção da gravidade de indisciplina entre professores e alunos não se distinguir significativamente, como constataram Rosado \& Januário (26), conjuntamente com a evidência de que existe uma el evada percepção da justiça da reacção do professor, reitera o entendimento de Doyle (8), quando $o$ autor refere que os alunos esperam que 0 professor estabeleça as regras, adaptando-se a elas com relativa facilidade.

\section{CONCLUSÕES}

O estudo destaca que, embora mais de metade dos al unos reproduza a totalidade da informação transmitida, uma elevada percentagem não relata a totalidade dessa informação, ou seja, existe, para um grande grupo de alunos, uma redução substantiva da informação transmitida. Esta constatação é relevante do ponto de vista da intervenção profissional já que sublinha a necessidade de determi nar modos de estruturar a informação passíveis de minimizar as perdas que decorrem entre a emissão e a recepção, compreensão e aceitação da informação. Por outro lado, a coerência da informação mostra estar inversamente relacionada tanto com a extensão da informação como com o número de ideias transmitidas. Tal revalida as prescrições didácticas que vêm sublinhando a importância da informação ser particularmente concisa e focada sobre as componentes críticas dos conteúdos de aprendizagem.

A reprodução de informação mostrou estar dependente, não só, da extensão e número de ideias transmitidas mas, também, do objectivo e forma dessa informação, sendo que a informação de carácter misto apresentou, sistematicamente, valores mais baixos de retenção. Este facto pode ser explicado devido à informação de carácter misto ser mais extensa e conter mais unidades de informação. Importa salientar, para o domínio da prática, que a utilização de estratégias instrucionais combinadas são benéficas quando não resultam em excesso de informação.

No tocante à influência das características dos alunos sobre a informação reproduzida e sobre a sua retenção, apenas o ano de escolaridade e a idade se fizeram sentir na variável extensão da informação, um indicador pouco relevante porquanto, como se constatou, é influenciado pela extensão da informação transmitida pelo professor. Na realidade, deve-se destacar que a maioria dos indicadores não foram influenciados pelas características dos alunos. Esta constatação real ça a importância da natureza da 
informação, na reprodução e retenção da informação, o que, mais uma vez, sublinha a importância da boa gestão da quantidade e qualidade da informação no processo de ensino-aprendizagem. Por outro lado, globalmente, os al unos consideraram que as reacções dos professores, em situação de regulação e controlo disciplinar, foram justas, fazendo supor níveis eleva dos de aceitação dessa informação. Considerando que o processo instrucional está intimamente dependente do seu efeito persuasivo, isto é, da sua aceitação por parte dos alunos, importa que os professores se preocupem com as questões da justiça percebida nas reacções aos comportamentos inapropriados, de modo a optimizar a sua intervenção.

Do ponto de vista das fragilidades conceptuais e metodológicas deste tipo de estudos importa destacar que indicadores baseados na extensão, número de ideias e densidade informativa reproduzida poderão não ser os mais indicados para estudar a retenção de informação, uma vez que não consideram os conteúdos concretos dessa informação mas apenas o seu volume, ou seja, a quantidade de informação, uma medida necessariamente limitada na compreensão deste fenómeno. Por outro lado, a simples análise da coerência não significa necessariamente que haja uma compreensão profunda das ideias transmitidas, porquanto a coerência pode ser aparente. Estudos sobre a compreensão da informação devem ser desenvolvidos ultrapassando a análise da simples memorização da informação. Importa, ainda, perce ber o grau de aceitação, da informação, pelos alunos, na medi da em que não basta retê-la e compreendêla sendo necessário que seja percebida como válida. Todavia, as conclusões da presente pesquisa reiteram a valência científica da linha de investigação centrada nos processos de retenção, compreensão e persuasão das mensagens instrucionais, desbravando caminho na identificação de variáveis mediadoras potenciadoras da eficácia pedagógica no âmbito das actividades desportivas.

\section{CORRESPONDÊNCIA}

\section{António Rosado}

Faculdade de Motricidade Humana

Estrada da Costa - Cruz Quebrada

1499 Lisboa Codex

Portugal

arosado@fmh.utl.pt 


\section{REFERÊNCIAS}

1. Alonso, B.(1988). La Disciplina Escolar en los Distintos M odelos Pedagógicos. Madrid: Ediciones Narcea.

2. Atkinson, R.; Shiffrin, R. (1971). The Control of Shortterm Memory. Scientific A merican, 225: 82-90. Maggill.

3. Breia, E., Rosado, A. (2004). Retenção de informação em episódios instrucionais típicos em treino desportivo. R evista Portuguesa de Ciências do D esporto 4, no 2, Setembro 2004 (suplemento)

4. Brito, M.(1986). Identificação dos E pisódios de Indisciplina em A ulas de Educação Física no Ensino Preparatório: A nálise do Comportamento de Professores e A lunos. Dissertação de Mestrado. Lisboa: ISEF-UTL.

5. Brunelle, J. ; Talbot, S. ;Achard, B. ; Tousignant, M. ; Berube,G. (1975). L'observation structuré de l'enseignement de l'éducation physique. M ouvement, 10(4): 195-209.

6. Cloes, M. ; Knoden, A. ; Pieron, M. (1991). Mémorisation d'information techniques reçues au cours dá activités sportives en situation contrôlée. In A ctes des IV Journées d' A utomne de I'ACAPS. Lille : Université de Lille, Vol. II, 222-223.

7. Cloes, M. ; Schmitz, C. ; Pieron, M. (1995). Utilisation de la technique du rappel stimulé dans I' analyse de la mémorisation du feedback par les participants, dans une situation d' enseignement simplifiée en volley-ball. In. CParé (Ed.), M ieux enseigner l' education physique ? Pensez-y ! Actes du séminaire international sur « La formation des enseignements à une pratique réflexine en èducation physique ». Trois-Rivières : Université du Québec à Trois Rivières, Dept. S.A.P. 294-264.

8. Doyle, W. (1981). Paradigmes de Recherche sur l'efficacité des Enseignants. In : Crahay, M. \& Lafontaine, D. (Eds). "L'A rt et la Science de L'Enseignement", Liège: Editions Labor, 435-481.

9. Estrela, M. T. (1986) Une Étude sur I'Indiscipline en Classe. Tese de Doutoramento. Lisboa: INIC

10. Estrela, M. T. (1995). Valores e Normatividade do Professor na Sala de Aula. Revista de Educação, 5(1): 65-73.

11. Estrela, M. T. (1996). Prevenção da Indisciplina e Formação de Professores. N oesis, 37 (Jan./Mar): 34-36.

12. Fenwick, D. T. (1998). Managing space, energy, and self: junior high teachers' experiences of classroom management. Teaching and Teacher Education, 14(6): 619-631.

13. Fields, B. A. (2000). School discipline: Is there a crisis in our schools? A ustralian Journal of Social Issues, 35: 73.

14. flavier, E, Hauw, D. Ria, L. \& Durand, M. (1999). emergence, Régulation et Signification des Conflits Professeur-Élèves au Cours de Leçons d'Éducation Physique: La Justice du Professeur. Besançon: Colloque AIESEP.

15. Fontana, D. (1986). La Disciplina en el Aula: Gestión y Control. Madrid: Ed. Santillana.

16. Gonçalves, A. (2000). A Indisciplina em Aulas de Educação Física: Percepções e Comportamentos de Professores e A lunos relativos à Indisciplina durante as Sessões de Ginástica ( solo) no 2ํ Ciclo do Ensino Básico. Dissertação de Mestrado. Lisboa: FMH.

17. Good, T. \& Brophy, J. (1978). Looking in Classroom. New York: Harper and Row Publishers.

18. Januário, N. (2002). R egulação e controlo disciplinar: percepção da informação e da justiça nas reacções dos professores por parte dos A lunos em Situações de Ensino. Dissertação de Mestrado. $\mathrm{FMH} / \mathrm{UTL}$

19. Kendler, H. (1972). Introdução à Psicologia. 2o vol. Lisboa: Fundação Calouste Gulbenkian.

20. Kennedy, E. (1982). Discipline in Physical Education Setting. The Physical Educator 39 (2): 91-94.

21. Marques da Costa, A. (1991). Estudo Qualitativo do "feedback" pedagógico - A nálise da Coerência entre a Informação do Professor e o Relato Posterior do Aluno. Dissertação de Mestrado. FMH/UTL.

22. Mendes, F.(1995). A Indisciplina em Aulas de Educação Física no 6.0 A no de Escolaridade: Contributo para o Estudo dos Comportamentos de Indisciplina e A nálise dos Procedimentos de Controlo U tilizados pelo Professor. Tese de Doutoramento. Porto : FCDEF-UP.

23. Piéron, M. \& Emonts, M.(1988). Analyse des Problèmes de Discipline dans les Classes d'Education Physique. Revue de l'Education Physique 28(1): 33-40.

24. Quina, J. (1993). A nálise da Informação evocada pelos A lunos em Aulas de Educação Física - U m Estudo sobre o "Feedback" Pedagógico. Dissertação de Mestrado. FMH/UTL.

25. Rosado, A. (1990). A disciplina nas Classes de Educação Física. H orizonte 7(38): 47-55.

26. Rosado, A., Januário, N. (1999). Perception des Incidents Disciplinaires. Etude de la variabilité de la perception d'incidents disciplinaires chez des élèves différenciés selon le sexe, I'âge, le groupe juvénile, la condition socioprofessionnelle des parents, les résultes scolaires et le groupe ethnique. Actas do Congresso da AIESEP, (Edição em CD-Rom). Besançon.

27. Rosado, A.; januário, n. (1999). Percepção de Incidentes Disciplinares entre grupos diferenciados de alunos pelo sexo, cultura juvenil, nível de sucesso escolar e grupo étnico". Trabalho de investigação indexado na EUDISED.

28. Sarmento, P.; Rosado, A.; Rodrigues, J.; Veiga, A. \& Ferreira, V. (1993). Pedagogia do D esporto - Instrumentos de O bservação Sistemática da Educação Física e D esporto ( $2^{\text {a }}$ ed.), Oeiras: Ediç̃̃es FMH.

29. Siedentop, D. (1983). D eveloping Teaching Skills in Physical Education ( $2^{a}$ ed.). Palo Alto: Mayfield Publishing Company.

30. Sulzer-azaroff, B. \& Mayer, G. (1977). A pplying Behavioranalysis Procedures with Children and Youth. Holt, Rinehart and Winston.

31. Supaporn, S., Dodds, P., \& Griffin, L. (2003). An ecological analysis of middle school misbehaviour through student and teacher perspectives. Journal of Teaching in Physical Education 22(3): 328-349. 